\title{
Cerebral Haemodynamics following Acute Ischaemic Stroke: Effects of Stroke Severity and Stroke Subtype
}

\author{
Osian Llwyd ${ }^{a} \quad$ Angela S.M. Salinet ${ }^{a}$ b Ronney B. Panerai ${ }^{a}$ \\ Man Y. Lam ${ }^{a}$ Nazia P. Saeed ${ }^{a}$ Fiona Brodie $^{a}$ Edson Bor-Seng-Shu ${ }^{b}$ \\ Thompson G. Robinson ${ }^{a}$ Ricardo C. Nogueira ${ }^{b}$ \\ ${ }^{a}$ Department of Cardiovascular Sciences Cerebral Haemodynamics in Ageing and Stroke \\ Medicine Research Group, University of Leicester, Leicester, UK; ${ }^{b}$ Neurology Department, \\ Hospital das Clinicas, School of Medicine, University of São Paulo, São Paulo, Brazil
}

\section{Keywords}

Blood flow velocity · Cerebral autoregulation - Acute stroke - Stroke subtype · Stroke severity

\section{Abstract}

Background: Acute ischaemic stroke (AIS) patients often show impaired cerebral autoregulation (CA). We tested the hypothesis that CA impairment and other alterations in cerebral haemodynamics are associated with stroke subtype and severity. Methods: AIS patients $(n=143)$ were amalgamated from similar studies. Data from baseline ( $<48 \mathrm{~h}$ stroke onset) physiological recordings (beat-to-beat blood pressure [BP], cerebral blood flow velocity (CBFV) from bilateral insonation of the middle cerebral arteries) were calculated for mean values and autoregulation index (ARI). Differences were assessed between stroke subtype (Oxfordshire Community Stroke Project [OCSP] classification) and severity (National Institutes of Health Stroke Scale [NIHSS] score $<5$ and 5-25). Correlation coefficients assessed associations between NIHSS and physiological measurements. Results: Thirty-two percent of AIS patients had impaired $C A(A R I<4)$ in affected hemisphere $(A H)$ that was similar between stroke subtypes and severity. $\mathrm{CBFV}$ in $\mathrm{AH}$ was comparable between stroke subtype and severity. In unaffected hemisphere $(\mathrm{UH})$, differences existed in mean CBFV between lacunar and total anterior circulation OCSP subtypes (42 vs. $56 \mathrm{~cm} \cdot \mathrm{s}^{-1}, p<0.01$ ), and mild and moderate-to-severe stroke severity ( $\left.45 \mathrm{vs} .51 \mathrm{~cm} \cdot \mathrm{s}^{-1}, p=0.04\right)$. NIHSS was associated with peripheral (diastolic and mean 
Llwyd et al.: Cerebral Haemodynamics following AIS

arterial BP) and cerebral haemodynamic parameters (CBFV and ARI) in the UH. Conclusions: AIS patients with different OCSP subtypes and severity have homogeneity in CA capability. Cerebral haemodynamic measurements in the UH were distinguishable between stroke subtype and severity, including the association between deteriorating ARI in UH with stroke severity. More studies are needed to determine their clinical significance and to understand the determinants of CA impairment in AIS patients.

(C) 2018 The Author(s)

Published by S. Karger AG, Basel

\section{Introduction}

Transcranial Doppler ultrasound has been widely used to evaluate cerebral haemodynamics in acute ischaemic stroke (AIS) patients, as it offers a non-invasive and real-time monitoring of cerebral blood flow velocity (CBFV). In particular, the assessment of cerebral autoregulation (CA), which reflects changes in $\mathrm{CBF}$ in relation to changes in blood pressure (BP), has provided further understanding of cerebral haemodynamic control [1]. Whilst previous studies have reported impairment of CA following acute stroke [2, 3], there are limited data on the effects of stroke subtype and severity. Cortical stroke appears to be associated with reduced $\mathrm{CA}$, more often in the affected hemisphere $(\mathrm{AH})$, whereas subcortical stroke may be associated with bilateral impairment $[4,5]$. Stroke severity may also be relevant, with moderate-to-severe stroke exhibiting greater CA impairment compared to minor stroke [6-9]. However, small sample size, and methodological heterogeneity in both data collection and analysis limit informed comparison between studies [10].

The Cerebral Haemodynamics in Ageing and Stroke Medicine (CHiASM) at the University of Leicester has a longstanding interest in studies of cerebral haemodynamic control mechanisms in the acute and recovery phases of AIS [7, 11-14]. Together with collaborators from the Hospital das Clinicas (São Paulo, Brazil), trained in cerebral haemodynamic assessment in Leicester, we have developed a large two-centre prospective database of AIS patients, utilising similar techniques in data acquisition and analysis that allow the interpretation of the effects of stroke severity and subtype on cerebral haemodynamic responses. We tested the hypothesis that stroke subtype or stroke severity significantly affects CA and consequently CBF precipitated by fluctuations in BP.

\section{Methods}

All investigators were trained at the same laboratory (University of Leicester, UK), and acquired physiological data to a standard data collection and analysis protocol. AIS patients admitted to the University Hospitals of Leicester NHS Trust (Leicester, UK), since 2007, or to the Hospital das Clinicas (São Paulo, Brazil), since 2015, and eligible for inclusion into ongoing studies of cerebral haemodynamics in AIS, were recruited and provided informed consent in compliance with local ethics committee approvals. All studies had common inclusion criteria: patients with clinical diagnosis of AIS, aged $\geq 18$ years, able to participate in study measurements, and informed consent; a common exclusion criterion was co-morbidity with life expectancy less than 3 months. In addition, patients were excluded from the database if: (a) missing key information (age, sex, and classification of stroke subtype according to Oxfordshire Community Stroke Project [OCSP] classification), (b) poor data quality, (c) duplicates arising from overlapping studies, (d) AIS classified as posterior circulation stroke, (e) physiological measurements performed after $48 \mathrm{~h}$ from stroke onset. 
The same protocol was used for all studies. Briefly, beat-to-beat BP was recorded continuously using the Finapres or Finometer devices (FMS, Finapres Measurement Systems, Arnhem, Netherlands). Heart rate (HR) was recorded using a 3-lead electrocardiogram, and end-tidal $\mathrm{CO}_{2}\left(\mathrm{etCO}_{2}\right)$ was measured via nasal prongs (Salter Labs) by an infrared capnograph (Capnocheck Plus and Transmai MX-200 in Leicester and São Paulo, respectively). Bilateral insonation of the middle cerebral arteries was performed using transcranial Doppler (Viasys Companion III; Viasys Healthcare and Doppler box, DWL for Leicester and São Paulo, respectively) with a 2-MHz probe, secured in place using a head-frame. Subjects were in a supine position. Systolic and diastolic BP was measured by brachial sphygmomanometry followed by 15 -min stabilisation and 5 -min baseline recording. When more than one baseline recording was available, the first acceptable recording was used for further analysis. Stroke subtype was defined by the OCSP classification; stroke severity was assessed by the NIHSS on hospital admission and at the time of cerebral haemodynamic assessment, with further categorisation by mild (NIHSS $\leq 5)$ [15] and moderate-to-severe stroke (NIHSS 6-25) [16].

\section{Data Analysis}

This has been described in detail previously [17]. In brief, data were simultaneously recorded onto a data acquisition system (PHYSIDAS, Department of Medical Physics, University Hospitals of Leicester) for subsequent off-line analysis. BP was calibrated at the start of each recording using systolic and diastolic values from brachial sphygmomanometry. The R-R interval was automatically marked from the electrocardiogram and mean BP and $\mathrm{CBFV}$ values were calculated for each cardiac cycle. If the etCO $\mathrm{C}_{2}$ recording was not consistent due to periods of breathing through the mouth, the highest et $\mathrm{CO}_{2}$ value was used.

Autoregulation index (ARI), which represents dynamic CA, was extracted by using the best least-squares fit between the CBFV step response and one of the 10 model ARI curves proposed by Tiecks et al. [18]. Values of ARI were only accepted for further analyses if strict conditions were met regarding the significance of the coherence between mean arterial BP CBFV and the normalised mean square error of fit to Tiecks model [19].

\section{Statistical Analysis}

Mean values of each variable were calculated from the entire baseline recording. Tests for normality were performed using the Shapiro-Wilk normality test. Data are presented as median and interquartile range (25th-75th percentile) and mean (SD) unless stated otherwise. Parameter values were considered outliers and excluded from further analysis if they were \pm 3 SD from the mean for all stroke patients. Differences between values derived from the AH or unaffected hemisphere (UH) were assessed with paired Student's $t$ test or the Wilcoxon test. Differences in variables between OCSP subtypes were assessed using a $\chi^{2}$ test, Fisher's exact test, ANOVA or Kruskal-Wallis test, followed by Tukey's or Dunn's multiple comparison test. Differences in variables between categorised NIHSS were assessed with Fisher's exact test, unpaired Student's $t$ test or Mann-Whitney test. Associations between variables were tested using linear regression correlation coefficient or Spearman's rank test as appropriate, where NIHSS was considered as a continuous variable. A $p$ value of $<0.05$ was defined as statistical significance.

\section{Results}

\section{Study Population}

Data for 143 AIS patients (105 Leicester; 38 São Paulo) of mean age 65 years (SD 13; 37\% female) with good quality baseline recordings were included. Key baseline characteristics are 
Table 1. Key baseline patient characteristics

\begin{tabular}{lcl}
\hline Variable & $n$ & \\
\hline Total patients & 143 & \\
Demographics & 53 & $(37)$ \\
$\quad$ Female & 143 & $65[13]$ \\
$\quad$ Age, years & & \\
\hline Patient history & 21 & $(19)$ \\
$\quad$ Smoker & 37 & $(33)$ \\
Ex-smoker & 31 & $(25)$ \\
Ischaemic heart disease & 22 & $(18)$ \\
Cerebrovascular disease & 18 & $(14)$ \\
Diabetes mellitus & 62 & $(50)$ \\
Hypertension & 57 & $(56)$ \\
Antihypertensive therapy & 29 & $(29)$ \\
Statin therapy & 23 & $(23)$ \\
Aspirin/antiplatelet/warfarin & 20 & $(24)$ \\
Ipsilateral ICA stenosis $>50 \%$ & 4 & $(5)$ \\
Contralateral ICA stenosis $>50 \%$ & & \\
\hline AIS features & 53 & $(37)$ \\
LACS & 66 & $(46)$ \\
PACS & 24 & $(17)$ \\
TACS & 117 & $19(5-30)$ \\
Onset to recording, h & 142 & $13: 00(11: 11-15: 30)$ \\
Time of recording, 24 h & 40 & $(49)$ \\
Thrombolysed & $70 / 73$ & $(49 / 51)$ \\
Ipsilateral side, R/L & 66 & $7(4-12)$ \\
Admission NIHSS & 122 & $5(3-10)$ \\
Assessment NIHSS & & \\
\hline
\end{tabular}

Data are presented as $n$ (\% of available data), mean [standard deviation] or median (interquartile range, 25-75th percentile), as appropriate. $n$, number of participants; ICA, internal carotid artery; AIS, acute ischaemic stroke; R, right; L, left; NIHSS, National Institutes of Health Stroke Scale; LACS, lacunar stroke syndrome; PACS, partial anterior circulation stroke syndrome; TACS, total anterior circulation stroke syndrome.

described in Table 1. Of these, 115 patients had bilateral middle cerebral artery recordings; 9 had unilateral recordings only of the AH and 19 only of the UH. Assessments of cerebral haemodynamic parameters were undertaken a median of $19 \mathrm{~h}$ (IQR 5-30) after stroke onset, with $49 \%$ of patients treated with intravenous thrombolysis.

\section{Influence of Stroke Subtype}

Patient characteristics and peripheral and cerebral haemodynamic data are described in Table 2 by each stroke subtype; lacunar (LACS, $n=53$ ), partial (PACS, $n=66$ ) and total anterior circulation infarcts (TACS, $n=24$ ). TACS patients were more likely to have a higher NIHSS and lower diastolic BP values at the time of haemodynamic assessment. With respect to differences in cerebral haemodynamic parameters between $\mathrm{AH}$ and $\mathrm{UH}$, systolic, mean and diastolic CBFV were significantly higher in the UH in the TACS patients only. Regarding the comparison between stroke subtypes for $\mathrm{AH}$ and $\mathrm{UH}$, only the UH had significant differences for systolic, mean and diastolic CBFV in the LACS compared to TACS patients, and additionally compared to PACS patients for diastolic CBFV alone. A poor goodness of fit to Tieck's model of CA was 
Table 2. Patient characteristics and peripheral and cerebral haemodynamic parameters by stroke subtype

\begin{tabular}{|c|c|c|c|c|c|c|c|}
\hline \multirow[t]{2}{*}{ Variable } & \multicolumn{2}{|c|}{ LACS } & \multicolumn{2}{|c|}{ PACS } & \multicolumn{2}{|c|}{ TACS } & \multirow[t]{2}{*}{$p$ value } \\
\hline & $n$ & & $n$ & & $n$ & & \\
\hline Demographics & 53 & & 66 & & 24 & & \\
\hline Female & 20 & $(38)$ & 22 & $(33)$ & 11 & $(46)$ & 0.55 \\
\hline Age, years & 53 & $67[12]$ & 66 & $65[14]$ & 24 & $60[15]$ & 0.13 \\
\hline \multicolumn{8}{|l|}{ AIS Features } \\
\hline Assessment NIHSS & 42 & $3(1-4)^{\ddagger}, \#$ & 57 & $5(3-9)^{\#}$ & 23 & $15(10-18)$ & $<0.001$ \\
\hline NIHSS $<5$ & 33 & (79) & 29 & $(51)$ & 3 & $(13)$ & $<0.001$ \\
\hline NIHSS 6-25 & 9 & (21) & 28 & (49) & 19 & (83) & $<0.001$ \\
\hline NIHSS $>25$ & 0 & & 0 & & 1 & $(4)$ & 0.19 \\
\hline Thrombolysed & 5 & $(23)$ & 24 & $(62)$ & 11 & $(52)$ & 0.01 \\
\hline Onset to recording, $\mathrm{h}$ & 41 & $22(14-36)$ & 53 & $15(3-30)$ & 23 & $16(6-28)$ & 0.12 \\
\hline \multicolumn{8}{|c|}{ Peripheral haemodynamic parameters } \\
\hline MABP, mm Hg & 53 & $102[16]$ & 66 & 99 [13] & 24 & 94 [11] & 0.08 \\
\hline Systolic BP, mm Hg & 53 & $149[23]$ & 66 & $144[23]$ & 24 & $142[16]$ & 0.36 \\
\hline Diastolic BP, mm Hg & $52^{\S}$ & $81[14]^{\#}$ & 66 & $77[12])$ & 24 & $72[12]$ & 0.03 \\
\hline $\mathrm{HR}$, beats/min & $52^{\S}$ & $71[10]$ & 66 & $71[13]$ & 24 & $75[14]$ & 0.24 \\
\hline etCO $_{2}, \mathrm{~mm} \mathrm{Hg}$ & 44 & $36[3]$ & 49 & $36[3]$ & 15 & $36[3]$ & 0.93 \\
\hline \multicolumn{8}{|c|}{ Cerebral haemodynamic parameters } \\
\hline Mean $\mathrm{CBFV}, \mathrm{cm} \bullet \mathrm{s}^{-1}-\mathrm{A}$ & 47 & $40[12]$ & $56^{\S}$ & $43[16]$ & 20 & $44[15]^{*}$ & 0.48 \\
\hline Mean $\mathrm{CBFV}, \mathrm{cm} \cdot \mathrm{s}^{-1}-\mathrm{U}$ & 50 & $42[13]^{\#}$ & 63 & $48[16]$ & 21 & $56[19]$ & $<0.01$ \\
\hline 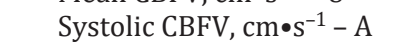 & 47 & $64[16]$ & $56^{\S}$ & $66[24]$ & 20 & $67[24]^{*}$ & 0.77 \\
\hline Systolic CBFV, $\mathrm{cm} \bullet \mathrm{s}^{-1}-\mathrm{U}$ & 50 & $67[18]^{\#}$ & 63 & $75[22]$ & 21 & $86[25]$ & $<0.01$ \\
\hline Diastolic CBFV, $\mathrm{cm} \bullet \mathrm{s}^{-1}-\mathrm{A}$ & 47 & $25[10]$ & $56^{\S}$ & $28[11]$ & 20 & $29[11]^{*}$ & 0.29 \\
\hline Diastolic CBFV, $\mathrm{cm} \cdot \mathrm{s}^{-1}-\mathrm{U}$ & 50 & $26[10]^{\ddagger, \#}$ & 63 & $31[12]$ & 21 & $36[13]$ & $<0.01$ \\
\hline ARI, arbitrary units - A & 46 & $4.6(3.7-6.0)$ & 51 & $4.6(3.6-6.2)$ & 18 & $4.2(3.6-5.4)$ & 0.82 \\
\hline ARI, arbitrary units - U & 48 & $4.4(3.7-6.0)$ & 56 & $4.8(4.1-6.1)$ & 17 & $4.1(3.4-5.3)$ & 0.20 \\
\hline $\mathrm{ARI}<4-\mathrm{A}$ & 15 & $(33)$ & 17 & $(33)$ & 5 & $(28)$ & 0.91 \\
\hline $\mathrm{ARI}<4-\mathrm{U}$ & 13 & (27) & 13 & $(23)$ & 8 & $(47)$ & 0.16 \\
\hline
\end{tabular}

Data are presented as $n$ (\% of available data), mean [standard deviation] or median (interquartile range, 25th-75th percentile), as appropriate. $p$ value, ANOVA or Kruskal-Wallis test between OCSP subtypes, followed by Tukey's or Dunn's multiple comparison test. $n$, number of participants; AIS, acute ischaemic stroke; NIHSS, National Institutes of Health Stroke Scale; MABP, mean arterial blood pressure; $\mathrm{HR}$, heart rate; etCO $\mathrm{C}_{2}$, end tidal $\mathrm{CO}_{2}$; $\mathrm{CBFV}$, cerebral blood flow velocity; ARI, autoregulation index; $\mathrm{A}$, affected hemisphere; $\mathrm{U}$, unaffected hemisphere; LACS, lacunar stroke syndrome; PACS, partial anterior circulation stroke syndrome; TACS, total anterior circulation stroke syndrome. ${ }^{*} p<0.05$ vs. unaffected side. ${ }^{\ddagger} p<0.05$ vs. PACS. ${ }^{\#} p<0.05$ vs. TACS. ${ }^{\S}$ Outlier removed from dataset.

found in 9 recordings from the $\mathrm{AH}$ and 13 from the $\mathrm{UH}$. These values were not included in further ARI analysis, giving a total of 115 and 121 sets of data, respectively, 99 of these bilateral. No differences in ARI between the AH and UH or between the stroke subtypes were observed, including the number that had ARI $<4$ that could be suggestive of impaired CA (Table 2) [20].

\section{Influence of Stroke Severity}

AIS patients were categorised into mild $(n=65)$ and moderate-to-severe $(n=56)$ stroke severity. Patient groups were of similar age and sex, with a significantly greater proportion of TACS and patients thrombolysed in the moderate-to-severe stroke group (Table 3). At the time of haemodynamic assessment, mild compared to moderate-to-severe stroke patients were more likely to have lower HR, and mean and diastolic CBFV in the UH; with haemodynamic assessment occurring later from stroke onset. With respect to differences in cerebral haemodynamic parameters between $\mathrm{AH}$ and $\mathrm{UH}$, systolic and diastolic CBFV in the UH was higher in the moderate-to-severe stroke population. There were no differences observed in 

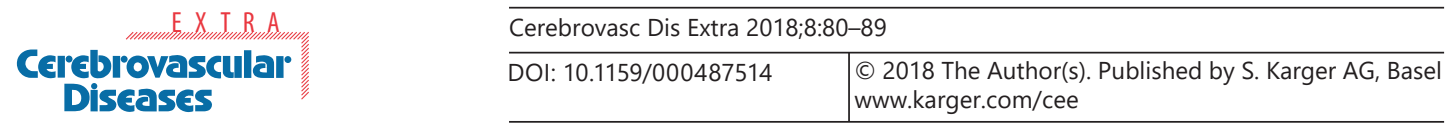

Llwyd et al.: Cerebral Haemodynamics following AIS

Table 3. Patient characteristics and peripheral and cerebral haemodynamic parameters by mild and moderate-to-severe stroke severity

\begin{tabular}{|c|c|c|c|c|c|}
\hline \multirow[t]{2}{*}{ Variable } & \multicolumn{2}{|c|}{$\begin{array}{l}\text { Mild stroke } \\
(\text { NIHSS } \leq 5)\end{array}$} & \multicolumn{2}{|c|}{$\begin{array}{l}\text { Moderate-to-severe stroke } \\
\text { (NIHSS 6-25) }\end{array}$} & \multirow[t]{2}{*}{$p$ value } \\
\hline & $n$ & & $n$ & & \\
\hline Demographics & 65 & & 56 & & \\
\hline Female & 26 & $(40)$ & 24 & (43) & 0.85 \\
\hline Age, years & 65 & 66 [12] & 56 & $64[14]$ & 0.45 \\
\hline \multicolumn{6}{|l|}{ AIS features } \\
\hline LACS & 33 & $(51)^{\ddagger}$ & 9 & (16) & $<0.001$ \\
\hline PACS & 29 & $(45)$ & 28 & (50) & 0.59 \\
\hline TACS & 3 & $(5)^{\ddagger}$ & 19 & (34) & $<0.001$ \\
\hline Thrombolysed & 11 & $(33)^{\ddagger}$ & 27 & $(60)$ & 0.02 \\
\hline Onset to recording, $\mathrm{h}$ & 61 & $21(11-31)^{\ddagger}$ & 53 & $10(3-27)$ & 0.03 \\
\hline \multicolumn{6}{|c|}{ Peripheral haemodynamic parameters } \\
\hline MABP, $\mathrm{mm} \mathrm{Hg}$ & 65 & $100[16]$ & 56 & 98 [13] & 0.52 \\
\hline Systolic BP, mm Hg & 65 & 145 [23] & 56 & $143[22]$ & 0.69 \\
\hline Diastolic BP, mm Hg & $64^{\S}$ & 79 [13] & 56 & $76[13]$ & 0.21 \\
\hline $\mathrm{HR}$, beats/min & 65 & $69[12]^{\dagger}$ & $55^{\S}$ & $74[11]$ & 0.03 \\
\hline etCO ${ }_{2}, \mathrm{~mm} \mathrm{Hg}$ & 52 & $35[3]$ & 35 & $36[3]$ & 0.38 \\
\hline \multicolumn{6}{|c|}{ Cerebral haemodynamic parameters } \\
\hline Mean $\mathrm{CBFV}, \mathrm{cm} \bullet \mathrm{s}^{-1}-\mathrm{A}$ & $58^{\S}$ & $41[13]$ & 46 & $43[17]$ & 0.40 \\
\hline Mean CBFV, $\mathrm{cm}^{-1} \mathrm{~s}^{-1}-\mathrm{U}$ & 59 & $45[14]^{\dagger}$ & 54 & $51[18]$ & 0.04 \\
\hline Systolic CBFV, $\mathrm{cm} \bullet \mathrm{s}^{-1}-\mathrm{A}$ & $58^{\S}$ & 64 [19] & 56 & $67[25]^{*}$ & 0.43 \\
\hline 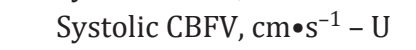 & 59 & $71[19]$ & 53 & $79[25]$ & 0.06 \\
\hline Diastolic CBFV, $\mathrm{cm}^{-1} \mathrm{~s}^{-1}-\mathrm{A}$ & $58^{\S}$ & $26[10]$ & 46 & $28[12]^{*}$ & 0.41 \\
\hline Diastolic CBFV, $\mathrm{cm} \cdot \mathrm{s}^{-1}-\mathrm{U}$ & 59 & $28[10]^{\ddagger}$ & 53 & 33 [13] & 0.02 \\
\hline ARI, arbitrary units - A & 53 & $5.0(3.8-6.3)$ & 43 & $4.3(3.6-5.9)$ & 0.27 \\
\hline ARI, arbitrary units - U & 54 & $5.1(4.1-6.3)$ & 46 & $4.4(3.9-5.2)$ & 0.06 \\
\hline $\mathrm{ARI}<4-\mathrm{A}$ & 15 & (28) & 14 & (33) & 0.66 \\
\hline ARI $<4-U$ & 12 & (22) & 13 & (28) & 0.50 \\
\hline
\end{tabular}

Data are presented as $n$ (\% of available data), mean [standard deviation] or median (interquartile range, 25th-75th percentile), as appropriate. $p$ value, Fisher's exact test, unpaired $t$ test or Mann-Whitney test between mild and moderate-tosevere stroke. $n$, number of participants; AIS, acute ischaemic stroke; NIHSS, National Institutes of Health Stroke Scale; MABP, mean arterial blood pressure; $\mathrm{HR}$, heart rate; et $\mathrm{CO}_{2}$, end tidal $\mathrm{CO}_{2}$; $\mathrm{CBFV}$, cerebral blood flow velocity; ARI, autoregulation index; A, affected hemisphere; U, unaffected hemisphere; LACS, lacunar stroke syndrome; PACS, partial anterior circulation stroke syndrome; TACS, total anterior circulation stroke syndrome. ${ }^{*} p<0.05$ vs. unaffected side. ${ }^{\ddagger} p<0.05$ vs. greater NIHSS. ${ }^{\S}$ Outlier removed from dataset.

ARI between the $\mathrm{AH}$ and $\mathrm{UH}$ or between mild and moderate-to-severe stroke patients, including those with ARI <4 (Table 3).

Stroke severity had a significant correlation with some peripheral (mean and diastolic BP) and cerebral haemodynamic parameters (Table 4). For every 4-point NIHSS score increase, there was an increase in the UH of $2.8,2.1$ and $1.7\left(\mathrm{~cm}^{\bullet} \mathrm{s}^{-1}\right)$ in systolic, mean and diastolic CBFV, respectively. In addition, a 4-point increase in NIHSS was associated with a 1.8 and $2.2 \mathrm{~mm} \mathrm{Hg}$ reduction in mean and diastolic BP or 0.3 reduction in ARI in the UH. However, no significant correlation with stroke severity was observed for cerebral haemodynamic parameters in the AH (Table 4). 
Table 4. Influence of stroke severity, assessed by NIHSS, on peripheral and cerebral haemodynamic parameters

\begin{tabular}{|c|c|c|c|c|}
\hline & $n$ & $\mathrm{CC}$ & Regression coefficient & $p$ value \\
\hline \multicolumn{5}{|l|}{ Peripheral parameters } \\
\hline MABP (mm Hg) & 122 & -0.1944 & $102-0.46 \bullet$ NIHSS & 0.03 \\
\hline Systolic BP (mm Hg) & 122 & -0.0096 & $147-0.39 \bullet$ NIHSS & 0.23 \\
\hline Diastolic BP (mm Hg) & 121 & -0.1505 & 82-0.56•NIHSS & $<0.01$ \\
\hline HR (beats/min) & 121 & 0.1312 & $69+0.26 \cdot$ NIHSS & 0.15 \\
\hline etCO ${ }_{2}(\mathrm{~mm} \mathrm{Hg})$ & 88 & 0.0771 & $35+0.04 \cdot$ NIHSS & 0.48 \\
\hline \multicolumn{5}{|l|}{ Cerebral haemodynamics } \\
\hline Mean CBFV $\left(\mathrm{cm}^{\circ} \mathrm{s}^{-1}\right)-\mathrm{A}$ & 104 & -0.005 & $42+0.013 \bullet$ NIHSS & 0.96 \\
\hline Mean CBFV $\left(\mathrm{cm}^{\prime} \mathrm{s}^{-1}\right)-\mathrm{U}$ & 113 & 0.1715 & $44+0.53 \bullet$ NIHSS & 0.04 \\
\hline Systolic CBFV $\left(\mathrm{cm} \cdot \mathrm{s}^{-1}\right)-\mathrm{A}$ & 104 & -0.009 & $65-0.03 \bullet$ NIHSS & 0.93 \\
\hline Systolic CBFV $\left(\mathrm{cm}^{\left.-\mathrm{s}^{-1}\right)}-\mathrm{U}\right.$ & 113 & 0.1873 & $70+0.70 \bullet$ NIHSS & $<0.05$ \\
\hline Diastolic CBFV $\left(\mathrm{cm}^{-\mathrm{s}^{-1}}\right)-\mathrm{A}$ & 104 & 0.0340 & $27+0.07 \cdot$ NIHSS & 0.73 \\
\hline Diastolic CBFV $\left(\mathrm{cm}^{-\mathrm{s}^{-1}}\right)-\mathrm{U}$ & 112 & 0.2147 & $28+0.43 \bullet$ NIHSS & 0.02 \\
\hline ARI (arbitrary units) - A & 96 & -0.1388 & $5.1-0.05 \bullet$ NIHSS & 0.18 \\
\hline ARI (arbitrary units) - U & 101 & -0.264 & $5.4-0.07 \bullet$ NIHSS & $<0.01$ \\
\hline
\end{tabular}

$p$ value, Pearson or Spearman's rank test as appropriate. $n$, number of participants; CC, correlation coefficient; MABP, mean arterial blood pressure; $\mathrm{HR}$, heart rate; et $\mathrm{CO}_{2}$, end tidal $\mathrm{CO}_{2}$; $\mathrm{CBFV}$, cerebral blood flow velocity; ARI, autoregulation index; A, affected hemisphere; U, unaffected hemisphere; NIHSS, National Institutes of Health Stroke Scale.

\section{Discussion}

This study demonstrated that overall many AIS patients had CA impairment, with an ARI below 4 during the first $48 \mathrm{~h}$ following stroke onset; $32 \%$ in the $\mathrm{AH}$ alone, $21 \%$ with bilateral impairment. There were no significant differences in ARI between stroke subtypes, defined by OCSP, or stroke severity, defined by NIHSS, except for a small reduction in ARI in the UH with increasing stroke severity. With respect to other measures of cerebral haemodynamics, no differences were observed for the AH by stroke subtype or severity. In the UH, TACS subtype and moderate-to-severe stroke severity was associated with increased CBFV.

\section{Influence of Stroke Subtype}

Few studies have evaluated the effect of stroke subtype on CA in the acute phase $[2,4,5]$, reporting bilateral impairment of CA with lacunar infarction and impaired CA predominantly in the AH in large vessel stroke $[4,5]$. Nonetheless, definitive conclusions are limited by small sample sizes and methodological differences, and the present study allowed changes to be assessed in a large cohort of AIS patients using comparable methods. Our data support differences in CBFV between cortical (TACS) and subcortical (LACS) stroke, interestingly in favour of increased CBFV in the UH in TACS. A number of possible explanations could be put forward for this. First, subcortical stroke may be associated with small vessel disease, caused by diabetes and chronic hypertension [4,5], and is more likely to lead to bilateral deterioration in cerebral haemodynamics [21]. Secondly, increased CBFV may be a response to the large vessel occlusion with recruitment of leptomeningeal collateral circulation from the UH. However, CBFV measured in the UH was similar to that reported in healthy individuals [17], and high levels of thrombolysis at $49 \%$ in the population studied may be associated with recanalization. Thirdly, CBFV in the UH may reflect cerebral steal, with there being previous 
Llwyd et al.: Cerebral Haemodynamics following AIS

reports in stroke patients where perfusion to the UH is augmented [22]. Finally, associated ICA stenosis may have resulted in previous changes in cerebral haemodynamics, particularly collateral blood flow, though only $20 \%$ of patients had significant ICA stenosis greater than $50 \%$, and all of these patients had different CBFV.

Nonetheless, despite CBFV changes, there was no significant effect of stroke subtype on $\mathrm{CA}$, as assessed by the ARI. This is in contrast to some of the previous literature [2, 4, 5]. Apart from the non-stationarity of dynamic CA [23], other possibilities for the disparity include: previous studies on AIS patients containing smaller sample sizes, from selected populations, different methodology to define stroke subtype [4], and differences in parameters adopted to quantify CA [10].

\section{Influence of Stroke Severity}

Minor stroke [9], smaller stroke volumes [3] and/or successful thrombolytic therapy [8] have been associated with no adverse impact on CA. Whereas large artery stroke [2] with associated increased severity [3], unsuccessful thrombolysis [9] or haemorrhagic transformation [24] may be associated with impaired CA, particularly during the first few days of admission, and have an adverse impact on neurological outcome [25]. The present study demonstrated an overall non-significant reduction in ARI in moderate-to-severe compared to mild stroke. This could be due to the haemodynamic assessment occurring on average 11 hours later in the mild stroke patients. However, the increasing NIHSS score was weakly, but significantly, associated with deteriorating ARI in the UH only. Furthermore, differences in CBFV related to stroke severity were also only observed in the UH.

Stroke severity may be associated with cardiovascular complications, including autonomic dysfunction [26]. Though there was increased sympathetic activation (as evidenced by HR) in patients with moderate-to-severe stroke severity, we found no correlation between HR and NIHSS. In addition, there may be more dependency on baroreceptor sensitivity when CA is impaired [27], which may be diminished when there is increased sympathetic activity and higher HR, as when patients suffer stroke of greater severity $[28,29]$. Finally, increased $\mathrm{BP}$ is well documented following stroke, though we found an inverse association between BP and NIHSS score, which is unsurprising in that more likely hypertensive LACS patients will have lower NIHSS scores. Nonetheless, our data should be interpreted with caution as there were fewer severe stroke patients included, and further studies of the effect of stroke severity on cerebral haemodynamics are warranted.

\section{Study Limitations}

There are a number of limitations to our study. First, though the OCSP is a widely used clinical classification, it does not consider underlying stroke aetiology, which may be more relevant in the potential impact on cerebral haemodynamics [30]. Secondly, NIHSS was not always taken on admission and at assessment, so maximum stroke severity could not be considered in further analysis. In addition, NIHSS was undertaken by several investigators. Thirdly, ARI was measured from spontaneous fluctuations between BP and CBF, though it is still to be determined whether small BP fluctuations are the best method for assessing CA [31]. The affected side had fewer good quality recordings and a poor goodness of fit to Tieck's model of CA, which could have added bias to some data sets. Finally, although, to our knowledge, this is currently the largest CA database of AIS patients to report cerebral haemodynamic changes, its size remains limited to definitely assess the effects of stroke subtype and severity. 


\section{Conclusion}

This large cohort demonstrated that cerebral haemodynamics represented by CBFV is significantly different according to stroke subtype and severity in the UH with higher values following TACS. This is a new finding that should be explored in future studies as a prognostic marker in this sub-population, particularly the impact of successful recanalization therapies. Our study corroborated previous findings that demonstrated an impairment of CA during the first $48 \mathrm{~h}$; with this large sample, we could demonstrate that this impairment is not influenced by stroke subtype and is poorly associated with stroke severity in the UH.

\section{Acknowledgments}

T.G.R. is a National Institute for Health Research Senior Investigator.

\section{Disclosure Statement}

The authors declare that they have no conflicts of interest.

\section{Funding Sources}

This study was supported by UK Engineering and Physical Sciences Research Council (grant No. EP/K041207/1) and São Paulo Research Foundation (FAPESP - N. 2014/04955-8 \& 2013/25953-0).

\section{References}

1 Panerai RB: Cerebral autoregulation: from models to clinical applications. Cardiovasc Eng 2008;8:42-59.

2 Xiong L, Tian G, Lin W, Wang W, Wang L, Leung T, Mok V, Liu J, Chen X, Wong K: Is dynamic cerebral autoregulation bilaterally impaired after unilateral acute ischemic stroke? J Stroke Cerebrovasc Dis 2017;26:10811087.

3 Castro P, Serrador JM, Rocha I, Sorond F, Azevedo E: Efficacy of cerebral autoregulation in early ischemic stroke predicts smaller infarcts and better outcome. Front Neurol 2017;8:6-10.

4 Immink RV, Van Montfrans G, Stam J, Karemaker JM, Diamant M, Van Lieshout JJ: Dynamic cerebral autoregulation in acute lacunar and middle cerebral artery territory ischemic stroke. Stroke 2005;36:2595-2600.

5 Guo Z-N, Liu J, Xing Y, Yan S, Lv C, Jin H, Yang Y: Dynamic cerebral autoregulation is heterogeneous in different subtypes of acute ischemic stroke. PLoS One 2014;9:e93213.

6 Reinhard M, Wihler C, Roth M, Harloff A, Niesen WD, Timmer J, Weiller C, Hetzel A: Cerebral autoregulation dynamics in acute ischemic stroke after rtPA thrombolysis. Cerebrovasc Dis 2008;26:147-155.

7 Atkins ER, Brodie FG, Rafelt SE, Panerai RB, Robinson TG: Dynamic cerebral autoregulation is compromised acutely following mild ischaemic stroke but not transient ischaemic attack. Cerebrovasc Dis 2010;29:228235.

8 Reinhard M, Rutsch S, Lambeck J, Wihler C, Czosnyka M, Weiller C, Hetzel A: Dynamic cerebral autoregulation associates with infarct size and outcome after ischemic stroke. Acta Neurol Scand 2012;125:156-162.

9 Chen J, Liu J, Xu W-H, Xu R, Hou B, Cui L-Y, Gao S: Impaired dynamic cerebral autoregulation and cerebrovascular reactivity in middle cerebral artery stenosis. PLoS One 2014; 9:e88232.

10 Meel-van den Abeelen ASS, Simpson DM, Wang LJY, Slump CH, Zhang R, Tarumi T, Rickards CA, Payne S, Mitsis GD, Kostoglou K, Marmarelis V, Shin D, Tzeng YC, Ainslie PN, Gommer E, Müller M, Dorado AC, Smielewski P, Yelicich B, Puppo C, Liu X, Czosnyka M, Wang CH, Novak V, Panerai RB, Claassen JAHR: Between-centre variability in transfer function analysis, a widely used method for linear quantification of the dynamic pressureflow relation: The CARNet study. Med Eng Phys 2014;36:620-627.

11 Saeed NP, Panerai RB, Horsfield M, Robinson TG: Does stroke subtype and measurement technique influence estimation of cerebral autoregulation in acute ischaemic stroke? Cerebrovasc Dis 2013;35:257-261. 
Llwyd et al.: Cerebral Haemodynamics following AIS

12 Salinet ASM, Robinson TG, Panerai RB: Cerebral blood flow response to neural activation after acute ischemic stroke: a failure of myogenic regulation? J Neurol 2013;260:2588-2595.

13 Salinet ASM, Panerai RB, Robinson TG: The longitudinal evolution of cerebral blood flow regulation after acute ischaemic stroke. Cerebrovasc Dis Extra 2014;4:186-197.

14 Panerai RB, Jara JL, Saeed NP, Horsfield MA, Robinson TG: Dynamic cerebral autoregulation following acute ischaemic stroke: comparison of transcranial Doppler and magnetic resonance imaging techniques. J Cereb Blood Flow Metab 2016;36:2194-2202.

15 Coutts SB, Dubuc V, Mandzia J, Kenney C, Demchuk AM, Smith EE, Subramaniam S, Goyal M, Patil S, Menon BK, Barber PA, Dowlatshahi D, Field T, Asdaghi N, Camden MC, Hill MD; for the TEMPO-1 Investigators: Tenecteplase-tissue-type plasminogen activator evaluation for minor ischemic stroke with proven occlusion. Stroke 2015;46:769-774.

16 Demaerschalk BM, Kleindorfer DO, Adeoye OM, Demchuk AM, Fugate JE, Grotta JC, Khalessi AA, Levy EI, Palesch YY, Prabhakaran S, Saposnik G, Saver JL, Smith EE; on behalf of the American Heart Association Stroke Council and Council on Epidemiology and Prevention: Scientific rationale for the inclusion and exclusion criteria for intravenous alteplase in acute ischemic stroke: a statement for healthcare professionals from the American Heart Association/American Stroke Association. Stroke 2016;47:581-641.

17 Patel N, Panerai RB, Haunton V, Katsogridakis E, Saeed NP, Salinet A, Brodie F, Syed N, D’Sa S, Robinson TG: The Leicester cerebral haemodynamics database: normative values and the influence of age and sex. Physiol Meas 2016;37:1485-1498.

18 Tiecks FP, Lam AM, Aaslid R, Newell DW: Comparison of static and dynamic cerebral autoregulation measurements. Stroke 1995;26:1014-1019.

19 Panerai RB, Haunton VJ, Hanby MF, Salinet ASM, Robinson TG: Statistical criteria for estimation of the cerebral autoregulation index (ARI) at rest. Physiol Meas 2016;37:1-17.

20 Caldas JR, Panerai RB, Haunton VJ, Almeida JP, Ferreira GSR, Camara L, Nogueira RC, Bor-Seng-Shu E, Oliveira ML, Groehs RRV, Ferreira-Santos L, Teixeira MJ, Galas FRBG, Robinson TG, Jatene FB, Hajjar LA: Cerebral blood flow autoregulation in ischemic heart failure. Am J Physiol -Regul Integr Comp Physiol 2017;312:R108-R113.

21 Guo Z-N, Xing Y, Wang S, Ma H, Liu J, Yang Y: Characteristics of dynamic cerebral autoregulation in cerebral small vessel disease: diffuse and sustained. Sci Rep 2015;5:15269.

22 Tsivgoulis G, Alexandrov AV: Cerebral hemodynamics in acute stroke: pathophysiology and clinical implications. J Vasc Interv Neurol 2008;1:65-69.

23 Panerai RB: Nonstationarity of dynamic cerebral autoregulation. Med Eng Phys 2014;36:576-584.

24 Castro P, Azevedo E, Serrador J, Rocha I, Sorond F: Hemorrhagic transformation and cerebral edema in acute ischemic stroke: link to cerebral autoregulation. J Neurol Sci 2017;372:256-261.

25 Santos GA, Petersen N, Zamani AA, Du R, Larose S, Monk A, Sorond FA, Tan CO: Pathophysiologic differences in cerebral autoregulation after subarachnoid hemorrhage. Neurology 2016;86:1950-1956.

26 Hilz MJ, Moeller S, Akhundova A, Marthol H, Pauli E, De Fina P, Schwab S: High NIHSS values predict impairment of cardiovascular autonomic control. Stroke 2011;42:1528-1533.

27 Tzeng Y-C, Lucas SJE, Atkinson G, Willie CK, Ainslie PN: Fundamental relationships between arterial baroreflex sensitivity and dynamic cerebral autoregulation in humans. J Appl Physiol 2010;108:1162-1168.

28 Robinson TG, Dawson SL, Eames PJ, Panerai RB, Potter JF: Cardiac baroreceptor sensitivity predicts long-term outcome after acute ischemic stroke. Stroke 2003;34:705-711.

29 Ogoh S, Tzeng Y-C, Lucas SJE, Galvin SD, Ainslie PN: Influence of baroreflex-mediated tachycardia on the regulation of dynamic cerebral perfusion during acute hypotension in humans. J Physiol 2010;588:365-371.

30 Asdaghi N, Jeerakathil T, Hameed B, Saini M, McCombe JA, Shuaib A, Emery D, Butcher K: Oxfordshire community stroke project classification poorly differentiates small cortical and subcortical infarcts. Stroke 2011;42:2143-2148.

31 Katsogridakis E, Bush G, Fan L, Birch AA, Simpson DM, Allen R, Potter JF, Panerai RB: Detection of impaired cerebral autoregulation improves by increasing arterial blood pressure variability. J Cereb Blood Flow Metab 2013;33:519-523. 